\title{
Application Value of Evidence-based Nursing in Patients with Multiple Myeloma and Evaluation of Nursing Quality
}

\author{
Zhu Xianglian, Wang Chunli \\ Department of Hematology, The First Affiliated Hospital of Jinan University, Guangzhou, China \\ Email address: \\ zhu20200821@163.com (Wang Chunli) \\ To cite this article: \\ Zhu Xianglian, Wang Chunli. Application Value of Evidence-Based Nursing in Patients with Multiple Myeloma and Evaluation of Nursing \\ Quality. American Journal of Nursing Science. Vol. 9, No. 5, 2020, pp. 354-358. doi: 10.11648/j.ajns.20200905.17
}

Received: August 23, 2020; Accepted: September 18, 2020; Published: September 28, 2020

\begin{abstract}
Objective to explore the application value and quality of evidence-based nursing in patients with multiple myeloma. Methods A total of 92 patients with multiple myeloma from December 2016 to December 2019 were selected as observation objects, and they were divided into control group 46 and observation group 46 according to the order of admission. The control group was given routine nursing care, and the observation group was given evidence-based nursing care. The bone pain relief rate, Pittsburgh Sleep Quality Score (PSQI), anxiety self-rating scale (SAS) score, depression self-rating scale (SDS) score and nursing quality were compared between the two groups. Results: The relief rate of bone pain in the observation group was higher than that in the control group $(\mathrm{P}<0.05)$. The quality of sleep, sleep time, sleep time, sleep efficiency, sleep disorders, daytime dysfunction scores and PSQI total scores in the observation group were lower than those in the control group after nursing $(\mathrm{P}<0.05)$. $\mathrm{P}<0.05)$; SAS and SDS scores in the observation group were lower than those in the control group after nursing $(\mathrm{P}<0.05)$. After the implementation of evidence-based nursing, the quality of basic nursing, specialist nursing, critical care, ward management, and health education nursing care of nursing staff was higher than that before implementation $(\mathrm{P}<0.05)$. Conclusion Applying evidence-based nursing to patients with multiple myeloma can improve bone pain relief, improve sleep quality, reduce anxiety and depression, and improve the quality of care.
\end{abstract}

Keywords: Evidence-based Nursing, Multiple Myeloma, Bone Pain, Sleep Quality, Psychological State, Nursing Quality

\section{Introduction}

Multiple myeloma is a malignant tumor that originates in plasma cells of bone marrow and produces monoclonal immunoglobulin. The incidence rate is about 1.0/100,000, accounting for $1.4 \%$ of all malignant tumors [1]. The etiology of the pathogenesis of multiple myeloma is not fully understood. Current clinical studies believe that it is closely related to occupational environment, radiation, smoking, drinking, drug factors, viral infections, etc [2-3]. Previous studies have shown that [4], Reasonable nursing measures can improve the quality and effect of bone pain and improve the quality of life of patients. In this study, the previous research foundation was used as an evidence-based basis to implement evidence-based nursing for patients with multiple myeloma and observe its clinical value and impact on the quality of care. The report is as follows.

\section{Materials and Methods}

\subsection{General Information}

92 patients with multiple myeloma from December 2016 to December 2019 in our hospital were selected as observation objects, and were divided into 46 cases in the control group and 46 cases in the observation group according to the admission order. Inclusion criteria: (1) 18-79 years old; (2) all received chemotherapy; (3) clear mind and independent judgment; (4) no communication barrier; (5) stable condition; (6) signed consent form. Exclusion criteria: (1) with malignant tumor; (2) with liver, lung, and kidney dysfunction; (3) history of mental illness; (4) acute cerebrovascular disease in the past month; (5) in the past month Major surgery; (6) Combining skin diseases. $\mathrm{n}$ the control group, there were 28 males and 18 females, aged 37-78 years old, with an average of $(62.76 \pm 6.95)$ 
years old; 19 cases of initial onset, 27 cases of relapse; classification: 23 cases of IgG type, 15 cases of IgA type, light chain type 7 There were 1 case of non-secretory type; ISS international staging: 8 cases in stage I, 23 cases in stage II, and 15 cases in stage III. In the observation group, there were 30 males and 16 females, aged 39-79 years, with an average age of (63.91 \pm 7.56$)$ years; 20 initial cases and 26 relapses; typing: 25 cases of IgG type, 14 cases of IgA type, and light chain type 6 There were 1 case of non-secretory type; ISS international stage: 9 cases in stage I, 24 cases in stage II, and 13 cases in stage III. The gender, age, type, and ISS international stage of the two groups were not significantly different $(\mathrm{P}>0.05)$, and were comparable.

\subsection{Method}

The control group adopts routine nursing: preaching disease knowledge to patients, instructing patients to use analgesics, closely observing changes in the disease and adverse reactions to medication, and managing complications symptomatically. The investigation team adopted evidence-based nursing: (1) to raise evidence-based questions: how to relieve patients' bone pain, improve sleep quality, Reduce negative psychology and improve the quality of care. (2) Establish evidence-based support: establish an evidence-based team, refer to the literature on multiple myeloma care and related research in platforms such as China HowNet, Wanfang, etc. to discuss the scientificity and feasibility of evidence-based evidence and determine Use evidence-based evidence to formulate nursing measures. Provide evidence-based nursing training and guidance to nursing staff to improve the overall team nursing level. (3) Evidence-based evidence: Related research [5] shows that there are three major problems in patients with multiple myeloma, (1) pain, urinary incontinence and other physiological experiences; (2) coping methods are mainly family support, increasing medical level, and self-regulation; Nursing measures include popularizing knowledge of multiple myeloma physiology experience, strengthening symptom management, improving information support and improving coping ability. (3) The patient's self-feeling burden comes from economic pressure, short recurrence period, and excessive psychological burden. Patients with multiple myeloma have moderate to severe pain and sleep disturbances. At the same time, related research [6] shows that the factors that affect the psychological resilience of patients with multiple myeloma include low monthly income per capita, rural cooperative medical payment methods, living in remote areas, less support, and habitual yielding coping; nursing measures are concerned about the existence of the above Factors, give patients appropriate guidance to improve their psychological resilience. Studies have shown that [7], the main adverse reactions of patients with multiple myeloma during treatment include nausea, decreased appetite, diarrhea and abdominal pain, bone marrow suppression, etc. The nursing measures mainly include detailed medication guidance, close observation of adverse reactions, and symptomatic treatment. In addition, studies have shown that increasing age, changes in blood uric acid and blood calcium can induce osteolytic destruction and pathological fractures [8]. (4) Nursing practice: (1) Bone pain care: Explain the cause of bone pain to the patient, encourage the patient to strengthen self-intervention, lightly massage the painful area with the finger belly or back of the finger, and rub it clockwise and counterclockwise. Infrared light is used to irradiate the painful area at a distance of $30-60 \mathrm{~cm}$, time $15-30 \mathrm{~min} /$ time, 1-2 times a day. Encourage the patient's family to help the patient massage [9]. Instruct patients to divert attention when pain occurs. Try to avoid patients participating in physical activities and physical weight-bearing. (2) Sleep care: rest on time, form regular sleep habits, maintain sufficient sleep time, start to sleep at 9:30 in the evening until wake up naturally. Before going to bed, defecate and defecate, soak your feet in warm water for $10-15 \mathrm{~min}$, and drink $100-150 \mathrm{ml}$ of warm water or milk. Take a comfortable posture while sleeping, and slowly and coordinately turn over. Instruct patients to relax their mind and body, eliminate distractions, regulate breathing, and ease exhalation and inhalation until they fall asleep. (3) Psychological Nursing: Nursing staff directly conduct psychological counseling to the patients, actively, enthusiastically, and concerned about the patient's condition, grasp the psychological dynamics, and give comfort, encouragement, persuasion, counseling, etc. through words and deeds. Encourage patients to take the initiative to confide, to relatives, friends, medical staff, etc., to vent bad emotions. Encourage the patient's family members to actively care about the patient's psychological state, and give counseling, care, and support. Instruct patients to adopt methods such as diverting attention, good memories, and self-suggestions to self-adjust their psychology. Strengthen health education for patients, provide timely information on the latest medical progress of diseases, and enhance patients' confidence in treatment. Try to reduce unnecessary expenditures of patients and reduce psychological pressure caused by economic burden. (4) Symptom management: Patients with gastrointestinal symptoms should be given correct dietary guidance, eat less and eat more meals, and eat 7-8 points each time. Drink $2500-3000 \mathrm{ml}$ of water every day to prevent constipation. After eating, massage the abdomen circularly for 15-20 minutes. Those with high blood uric acid and blood calcium levels are encouraged to promote uric acid excretion through multiple factors, and eat less calcium-rich and high-purine foods, such as shrimp skin, kelp, soybeans, lentils, onions, etc, to avoid alcoholism.

\subsection{Evaluation Criteria}

(1) Relief of bone pain: Grade 1: no pain; Grade 2: bone pain but tolerable; Grade 3: pain is unbearable, sleep is affected, strong analgesics are required. Significant relief: bone pain reduction $\geq 2$ grades; partial relief: bone pain reduction 1 grade; no relief: no significant relief of bone pain, temporary relief and restitution or even worsening. Bone pain relief rate $=$ (significant relief + partial relief) $/ 46 \times 100 \%$. (2) Comparison of sleep quality between two groups: Pittsburgh Sleep Quality Index Scale (PSQI) was used for evaluation, including sleep quality ( $0-3$ points), time to fall asleep ( $0-3$ points), sleep time 
(0-3 points), Sleep efficiency (0-3 points), sleep disorders (0-3 points), hypnotic drugs (0-3 points), daytime dysfunction (0-3 points) score and total PSQI score (0-15 points), score The higher the sleep quality, the worse. (3) Psychological state: The psychological state of patients was assessed using the Self-Assessment Scale of Anxiety (SAS) and Self-Assessment of Depression (SDS) compiled by William WK Zung in the United States, with a total score of 100 points. The higher the score, the greater the degree of anxiety and depression Heavier. (4) Compare the quality of evidence-based nursing before and after implementation, including basic nursing, specialist nursing, critical patient nursing, ward management, and health education, each with a score of 100 .

\subsection{Statistical Analysis}

Using SPSS19.0 statistical software analysis, measurement data is expressed by $\pm \mathrm{s}$, $\mathrm{t}$ test is performed, count data is expressed by $\%, \chi 2$ test is performed, and $\mathrm{P}<0.05$ indicates statistical significance.

\section{Results}

\subsection{Comparison of Relief of Bone Pain Between the Two Groups}

The bone pain relief rate of the observation group was higher than that of the control group $(\mathrm{P}<0.05)$, see Table 1 .

Table 1. Comparison of bone pain relief rate between the two groups [n (\%)].

\begin{tabular}{|c|c|c|c|c|c|}
\hline Group & $\mathbf{n}$ & Significant relief & Partial relief & Not relieved & Remission rate \\
\hline Observation group & 46 & $13(28.26)$ & $25(54.35)$ & $8(17.39)$ & 82.61 \\
\hline Control group & 46 & $6(13.04)$ & $21(45.65)$ & $19(41.30)$ & 58.70 \\
\hline$\chi^{2}$ & & & & & 6.343 \\
\hline$P$ & & & & & 0.012 \\
\hline
\end{tabular}

\subsection{Comparison of PSQI Sleep Quality Score Between Two Groups}

There were no significant differences in the scores of sleep quality, time to fall asleep, sleep time, sleep efficiency, sleep disorders, daytime dysfunction scores and total PSQI scores of the two groups before nursing $(\mathrm{P}>0.05)$. The scores of the observation group after nursing were lower than those of the control group $(\mathrm{P}<0.05)$, see Table 2 .

Table 2. Comparison of PSQI sleep quality scores between two groups $( \pm s$, points).

\begin{tabular}{|c|c|c|c|c|c|c|c|c|}
\hline \multirow{2}{*}{ PSQI } & \multicolumn{2}{|l|}{ Before nursing } & \multirow{2}{*}{$t$} & \multirow{2}{*}{$\boldsymbol{P}$} & \multicolumn{2}{|l|}{ After care } & \multirow{2}{*}{$t$} & \multirow{2}{*}{$\boldsymbol{P}$} \\
\hline & Observation group & Control group & & & Observation group & Control group & & \\
\hline Sleep quality & $1.75 \pm 0.29$ & $1.76 \pm 0.32$ & 0.157 & 0.876 & $0.88 \pm 0.23$ & $1.27 \pm 0.28$ & 7.300 & 0.000 \\
\hline Time to fall asleep & $1.62 \pm 0.35$ & $1.65 \pm 0.38$ & 0.394 & 0.695 & $0.71 \pm 0.20$ & $1.06 \pm 0.25$ & 7.415 & 0.000 \\
\hline sleeping time & $1.74 \pm 0.30$ & $1.71 \pm 0.37$ & 0.427 & 0.670 & $0.79 \pm 0.11$ & $1.23 \pm 0.21$ & 12.588 & 0.000 \\
\hline Sleep efficiency & $1.77 \pm 0.32$ & $1.79 \pm 0.39$ & 0.269 & 0.789 & $1.01 \pm 0.19$ & $1.38 \pm 0.29$ & 7.238 & 0.000 \\
\hline sleep disorder & $1.72 \pm 0.28$ & $1.70 \pm 0.36$ & 0.297 & 0.767 & $0.73 \pm 0.13$ & $1.04 \pm 0.24$ & 7.703 & 0.000 \\
\hline $\begin{array}{l}\text { Daytime } \\
\text { dysfunction }\end{array}$ & $2.06 \pm 0.37$ & $2.10 \pm 0.33$ & 0.547 & 0.586 & $1.04 \pm 0.15$ & $1.47 \pm 0.30$ & 8.695 & 0.000 \\
\hline Total score & $12.05 \pm 2.22$ & $12.08 \pm 2.47$ & 0.061 & 0.951 & $5.74 \pm 1.13$ & $8.30 \pm 1.83$ & 8.073 & 0.000 \\
\hline
\end{tabular}

\subsection{Comparison of Mental State Between the Two Groups}

There was no statistically significant difference in SAS and SDS scores between the two groups before nursing (P $>0.05$ ), and the scores of the observation group after nursing were lower than those of the control group $(\mathrm{P}<0.05)$, see Table 3 .

Table 3. Comparison of mental state between two groups ( \pm s, points).

\begin{tabular}{|c|c|c|c|c|c|}
\hline \multirow{2}{*}{ Group } & \multirow{2}{*}{$\mathbf{n}$} & \multicolumn{2}{|l|}{ SAS } & \multicolumn{2}{|l|}{ SDS } \\
\hline & & Before nursing & After care & Before nursing & After care \\
\hline Observation group & 46 & $62.34 \pm 6.37$ & $37.46 \pm 5.53$ & $65.93 \pm 7.19$ & $38.67 \pm 4.23$ \\
\hline Control group & 46 & $61.91 \pm 5.86$ & $50.39 \pm 5.26$ & $66.80 \pm 6.92$ & $49.50 \pm 5.65$ \\
\hline$t$ & & 0.337 & 23.967 & 0.591 & 10.407 \\
\hline$P$ & & 0.737 & 0.000 & 0.556 & 0.000 \\
\hline
\end{tabular}

\subsection{Comparison of Nursing Quality Before and After Implementation of Evidence-based Nursing}

After implementing evidence-based nursing, the basic nursing, specialist nursing, critical patient nursing, ward management, 
and health education nursing quality assessment were higher than before implementation $(\mathrm{P}<0.05)$, see Table 4 .

Table 4. Comparison of nursing quality before and after implementing evidence-based nursing ( $\pm s$, points, number of nursing staff: $n=22)$.

\begin{tabular}{llllll}
\hline time & Basic nursing & Specialist nursing & Critical care & Ward management & Health education \\
\hline Before implementation & $90.12 \pm 3.75$ & $90.87 \pm 4.26$ & $88.02 \pm 5.21$ & $87.29 \pm 4.03$ & $85.71 \pm 4.07$ \\
After implementation & $95.33 \pm 3.81$ & $95.01 \pm 3.39$ & $93.97 \pm 4.32$ & $92.32 \pm 5.26$ & $96.13 \pm 3.25$ \\
$t$ & 4.571 & 3.567 & 4.124 & 3.561 & 9.356 \\
$P$ & 0.000 & 0.001 & 0.000 & 0.001 & 0.000 \\
\hline
\end{tabular}

\section{Discussion}

Multiple myeloma mortality accounts for $1.9 \%$ of all cancer deaths [10]. In recent years, with the development of medicine, the level of treatment has been continuously improved, but it is still difficult to cure. Patients with multiple myeloma require long-term treatment. Under the influence of various factors such as disease distress, economic pressure, and role change, the patient's life treatment is seriously affected [11].

Evidence-based nursing guides the care of patients with multiple myeloma by searching for previous studies, which makes the care more reasonable and scientific, and transforms the traditional casual and empirical routine care. The results of this study showed that the bone pain relief rate of the observation group was higher than that of the control group. It is suggested that evidence-based nursing can improve the effect of bone pain relief in patients with multiple myeloma. Analysis of the reason may be evidence-based nursing by looking for the causes and nursing experience of bone pain in patients with multiple myeloma, and applying it to the care of patients with bone pain Medium, improve the effect of bone pain care. The results of this study showed that the sleep quality, time to fall asleep, sleep time, sleep efficiency, sleep disorders, daytime dysfunction scores and total PSQI scores of the observation group after nursing were lower than those of the control group. It is suggested that evidence-based nursing can improve the sleep quality of patients with multiple myeloma. The reason for analysis is that evidence-based nursing implements care by combining the factors affecting the sleep quality of patients, so that the nursing measures are more targeted to sleep quality, which is conducive to improving the sleep of patients quality. This study showed that the SAS and SDS scores of the observation group after nursing were lower than those of the control group. It is suggested that evidence-based nursing can reduce the anxiety and depression mental state of patients with multiple myeloma. The reason for analysis is that evidence-based nursing affects the related factors of patients' psychological resilience through intervention, and eliminates or alleviates the psychological impact of unfavorable factors, such as direct comfort and encouragement, helping patients fight for social support, etc, which is conducive to alleviating adverse psychological states [12-14]. After the implementation of evidence-based nursing, the basic nursing, specialist nursing, critical care, ward management, and health education nursing quality evaluation are higher than before implementation. Analyze the reasons, and evidence-based nursing integrates real and reliable scientific evidence into nursing practice Combined with the personal experience and skills of nursing staff, the traditional narrow nursing model is transformed into a new nursing model based on scientific research results, which improves the quality of nursing [15].

\section{Conclusions}

In summary, evidence-based nursing applied to patients with multiple myeloma can improve the relief effect of bone pain, improve sleep quality, reduce anxiety and depression mental state, and improve the quality of care.

\section{References}

[1] Zhao Wei, Xu Qin, Xie Jun. The application of home cognitive behavior therapy in cancer-induced fatigue of patients with multiple myeloma chemotherapy [J]. Anhui Medicine, 2019, 23 (03): 571-574.

[2] Kathleen Colson. Treatment-related symptom management in patients with multiple myeloma: a review [J]. Supportive Care in Cancer, 2015.

[3] Yun, Eun, Kyoung, et al. EFFECTS OF STRESS APPRAISAL ON THE QUALITY OF LIFE OF KOREAN ADULT PATIENTS AND THEIR PRIMARY FAMILY CAREGIVERS WITH MULTIPLE MYELOMA [J]. Oncology Nursing Forum, 2015.

[4] Li Yanyun. Advances in nursing of bone pain in patients with multiple myeloma [J]. Shanghai Nursing, 2015, 15 (07): 147.

[5] Sun Bowei, Fu Rong, Chu Hong, et al. Qualitative research on the true experience of multiple myeloma patients during treatment [J]. Chinese Journal of Practical Nursing, 2018, 34 (31): 2445-2450.

[6] Sun Bowei, Fu Rong, Chu Hong, et al. Analysis of the status and influencing factors of psychological elasticity in 144 patients with multiple myeloma [J]. Journal of Nursing, 2019, 26 (10): 38-43.

[7] Zhao Rong, Yang Shuang. Nursing care of patients with multiple myeloma treated by subcutaneous injection of bortezomib [J]. Chinese Journal of Practical Nursing, 2015, 31 (02): 37-38.

[8] Hao Jinghong. Analysis of related factors and nursing of patients with multiple myeloma complicated with bone abnormalities [J]. Chinese Journal of Modern Nursing, 2016, 22 (12): 1680-1682. 
[9] Tang Xiaoxia, Zhou Zeping, Bi Hui, et al. Investigation and analysis of thrombosis prevention in patients with multiple myeloma [J]. Anhui Medicine, 2019, 23 (02): 395-398.

[10] Wang Deyin, Hao Yunliang, Xiao Meng, et al. Epidemiology and etiology analysis of multiple myeloma [J]. International Journal of Epidemiology and Infectious Disease, 2018, 45 (04): 277-280.

[11] Chen Yashu, Liu Min, Xu Xiaoxu, et al. Analysis of risk factors for survival of patients with multiple myeloma [J]. PLA Journal of Preventive Medicine, 2019, 37 (07): 78-79.

[12] Nursing C O, Graduate School of Seoul National University, Seoul, et al. Factors Influencing Quality of Life in Multiple Myeloma Patients [J]. Asian Oncology Nursing, 2015.
[13] Ling L. Effect of evidence-based nursing intervening in prevention of toxic and adverse effects in multiple myeloma patients with chemotherapy [J]. Journal of Clinical Medicine in Practice, 2017.

[14] Jo K S, Ban J Y, Yoon J Y, et al. Quality of Life in Patients with Multiple Myeloma [J]. Korean Journal of Adult Nursing, 2016, 28 (3).

[15] MA Yu-ting. Self management and nursing intervention in patients with multiple myeloma [J]. Journal of Anhui Health Vocational \& Technical College, 2016. 